\title{
Propagation of S-waves in a non-homogeneous anisotropic incompressible and initially stressed medium
}

\author{
S. Gupta ${ }^{1 *}$, S. Kundu' ${ }^{1}$ A.K. Verma ${ }^{2}$ and R. Verma ${ }^{3}$ \\ ${ }^{1 *}$ Department of Applied Mathematics, I.S.M. Dhanbad, INDIA \\ ${ }^{2}$ Department of Mathematics, Hampton University, Hampton, VA, USA \\ ${ }^{3}$ Department of Mathematics, Norfolk State University, Norfolk, VA, USA \\ *Corresponding Author: e-mail: shishir_ism@yahoo.com, Tel +91-326-2235464
}

\begin{abstract}
In this paper we have studied the propagation of shear waves in a non-homogeneous anisotropic incompressible and initially stressed medium. Analytical analysis reveals that the velocities of the shear waves depend upon the direction of propagation, the anisotropy, the non-homogeneity of the medium and the initial stress. Numerical computation shows that the presence of initial compressive stress in the medium reduces the velocity of propagation whereas, the tensile stress increases it. It is found that the variation in parameters associated with anisotropy and non-homogeneity of the medium directly affects the velocity of the wave. The velocity of wave also depends on the inclination of the direction of its propagation. An increase in the inclination angle decreases the velocity in the beginning and takes a minimum value before increasing.
\end{abstract}

Keywords: Shear waves; anisotropic; stress; non-homogeneity; half-spaces.

\section{Introduction}

The term "Initial stress" is meant by stresses developed in a medium before it is being used for study. The earth is an initially stressed medium, Due to presence of external loading, slow process of creep and gravitational field, considerable amount of stresses (called pre-stresses or initial stresses) remain naturally present in the layers. These stresses may have significant influence on elastic waves produced by earthquake or explosions and also in the stability of the medium. The propagation of surface waves is well documented in the literature (e.g., Achenbach (1973), Bath (1968), Biot (1965), Ewing (1957)). Biot (1940) formulated the dynamical equations for pre-stressed elastic medium and discussed the influence of pre-stresses on the propagation of elastic waves in a body.

The problem of finite deformations of an elastic body and the effect of high initial stress on wave propagation were discussed in a series of investigations by Kappus (1939), Murnaghan (1951) and others. Qian et al. (2004) have investigated the effect of initial stress on the propagation behavior of SH-waves in multilayered piezoelectric composite structures. Chattopadhyay et al. (2009) have studied the propagation of shear waves in an internal magnetoelastic monoclinic stratum sandwiched between two semiinfinite isotropic elastic media and with a rectangular irregularity in lower interface. Chattopadhyay et al. (2010) have also investigated the propagation of shear waves in a monoclinic layer with an irregularity lying between two isotropic semi infinite elastic medium. The effect of inhomogeneous initial stress on Love wave propagation in layered magneto-electro-elastic structures have been studied by Zhang et al. (2008). Sharma (2005) has demonstrated the effect of initial stress on the propagation of plane waves in a general anisotropic poro-elastic medium.

The Edge wave propagation in an incompressible anisotropic initially stressed plate of finite thickness has been studied by Dey et al. (2009). Addy et al. (2005) have studied Rayleigh waves in a viscoelastic half-space under initial hydrostatic stress in presence of the temperature field. Liu et al. (2008) have demonstrated the propagation characteristics of converted refracted wave and its application in static correction of converted wave. Moczo et al. (2007) provided mathematical modeling of seismic wave propagation using the Finite-Difference time-domain method. Huber (2010) has explained the physical meaning of a nonlinear evolution equation of the fourth order relating to locally and non-locally supercritical waves in his work. Duan1 et al. $(2006,2007)$ 
have investigated heterogeneous fault stresses from previous earthquakes and the effect on dynamics of parallel strike-slip faults and non-uniform pre-stress from prior earthquakes and the effect on dynamics of branched fault systems.

Zhou and Chen (2005) have studied the influence of seismic cyclic loading history on small strain shear modulus of saturated sands. Sharma et al. (2007) discussed about the wave velocities in a pre-stressed anisotropic elastic medium. Selim et al. (2006) have demonstrated the propagation and attenuation of seismic body waves in dissipative medium under initial and couple stresses. Seismology is the study of progressive elastic wave. But most of this studies and investigations do not include very important factor viz, the influence of initial stress, anisotropy and non-homogeneity present in the body. In this paper, an attempt has been made to show the effect of initial stress, the anisotropy and non-homogeneity of the medium on the propagation of shear wave.

\section{Solution of the problem}

Most materials behave as incompressible media and their influence on seismic waves are very high. (The velocities of longitudinal waves in them are very high) The varieties of hard rocks present in the earth are also, almost incompressible. Due to the factors like external pressure, slow process of creep, difference in temperature, manufacturing processes, nitriding, pointing etc., the medium stay under high stresses. These stresses are regarded as initial stresses. Owing to the variation of elastic properties and the presence of these initial stresses, the medium becomes anisotropic as well.

We consider an unbounded incompressible anisotropic medium under initial stresses $\mathrm{S}_{11}$ and $\mathrm{S}_{22}$ along the $x$-, $y$ - directions respectively. When the medium is slightly disturbed, the incremental stresses $s_{11}, s_{12}$ and $s_{22}$ are developed and the equations of motion given by Biot (1965) are

$$
\begin{aligned}
& \frac{\partial s_{11}}{\partial x}+\frac{\partial s_{12}}{\partial y}-P \frac{\partial w}{\partial y}=\rho \frac{\partial^{2} u}{\partial t^{2}} \\
& \frac{\partial s_{12}}{\partial x}+\frac{\partial s_{22}}{\partial y}-P \frac{\partial w}{\partial x}=\rho \frac{\partial^{2} v}{\partial t^{2}} .
\end{aligned}
$$

where $P=\mathrm{S}_{22}-\mathrm{S}_{11}, w=\frac{1}{2}\left(\frac{\partial v}{\partial x}-\frac{\partial u}{\partial y}\right)$, and $\rho$ represents the density of the medium. Also $s_{i \mathrm{j}}$, are incremental stresses, $(u, v)$ are incremental deformations, $w$ is the rotational component about the $z$-axis.

The incremental stress-strain relations for an incompressible medium may be taken as

$$
s_{11}-s=2 N e_{x x}, s_{22}-s=2 N e_{y y} \text { and } s_{12}=2 Q e_{x y} .
$$

where $s=\frac{S_{11}+S_{22}}{2}, e_{i \text { j }}$ are incremental strain components and $N$ and $Q$ are the rigidities of the medium.

The incompressibility condition $e_{x x}+e_{y y}=0$, is satisfied by:

$$
u=-\frac{\partial \varphi}{\partial y} \text { and } v=\frac{\partial \varphi}{\partial x} \text {. }
$$

Using eqs. (3) and (4) in eqs. (1) and (2), we obtain

$$
\begin{aligned}
& \frac{\partial s}{\partial x}-\frac{\partial}{\partial y}\left[\left(2 N-Q-\frac{P}{2}\right) \frac{\partial^{2} \varphi}{\partial x^{2}}+\left(Q+\frac{P}{2}\right) \frac{\partial^{2} \varphi}{\partial y^{2}}\right]=\rho \frac{\partial^{2} u}{\partial t^{2}}, \\
& \frac{\partial s}{\partial y}+\frac{\partial}{\partial x}\left[\left(2 N-Q-\frac{P}{2}\right) \frac{\partial^{2} \varphi}{\partial y^{2}}+\left(Q-\frac{P}{2}\right) \frac{\partial^{2} \varphi}{\partial x^{2}}\right]=\rho \frac{\partial^{2} v}{\partial t^{2}} .
\end{aligned}
$$

We assume the non-homogeneity as

$$
\left.\begin{array}{l}
Q=Q_{0}(1+a y) \\
N=N_{0}(1+b y) \\
\rho=\rho_{0}(1+c y)
\end{array}\right\}
$$

where $N_{0}$ and $Q_{0}$ are rigidities and $\rho_{0}$ is the density in homogeneous isotropic medium. Eliminating $s$ from eqs. (5) and (6) we get 


$$
\begin{aligned}
& {\left[Q_{0}(1+a y)-\frac{P}{2}\right] \frac{\partial^{4} \varphi}{\partial x^{4}}+\left[4 N_{0}(1+b y)-2 Q_{0}(1+a y)\right] \frac{\partial^{4} \varphi}{\partial x^{2} \partial y^{2}}} \\
& +\left[Q_{0}(1+a y)+\frac{P}{2}\right] \frac{\partial^{4} \varphi}{\partial y^{4}}+\left(4 N_{0} b-2 Q_{0} a\right) \frac{\partial^{3} \varphi}{\partial x^{2} \partial y}+2 Q_{0} a \frac{\partial^{3} \varphi}{\partial y^{3}} \\
& =\rho_{0}(1+c y)\left(\frac{\partial^{4} \varphi}{\partial x^{2} \partial t^{2}}+\frac{\partial^{4} \varphi}{\partial y^{2} \partial t^{2}}\right)+\rho_{0} c \frac{\partial^{3} \varphi}{\partial y \partial t^{2}} .
\end{aligned}
$$

\section{Solution of the problem}

For propagation of sinusoidal waves in any arbitrary direction we take the solution of eq.(8) as

$$
\varphi(x, y, t)=A e^{i k\left(p_{1} x+p_{2} y-c_{1} t\right)} .
$$

where $p_{1}$ and $p_{2}$ are cosine of the angles made by the direction of propagation with the $x$-and $y$-axes, and $c_{1}$ and $k$ are the velocity of propagation and the wave number respectively.

Using eq.(9) in eq.(8) and equating real and imaginary parts separately, one gets

$$
\begin{aligned}
\left(\frac{c_{1}}{\beta}\right)^{2}= & \frac{1}{(1+c y)}\left\{\left(1+a y-\frac{P}{2 Q_{0}}\right) p_{1}^{4}+2\left[\frac{2 N_{0}}{Q_{0}}(1+b y)-(1+a y)\right] p_{1}^{2} p_{2}^{2}\right. \\
& \left.+\left(1+a y+\frac{P}{2 Q_{0}}\right) p_{2}^{4}\right\},
\end{aligned}
$$

and

$$
\left(\frac{c_{1}}{\beta}\right)^{2}=2\left(\frac{2 N_{0}}{Q_{0}} \frac{b}{c}-\frac{a}{c}\right) p_{1}^{2}+2\left(\frac{a}{c}\right) p_{2}^{2}
$$

where $\beta=\left(\frac{Q_{0}}{\rho_{0}}\right)^{\frac{1}{2}}$, the velocity of shear wave in homogeneous isotropic medium. Eq. (10) gives the velocity of propagation of shear wave and eq. (11) gives the damping.

Equation (10) shows that the velocity depends on the anisotropy factor $\left(\frac{N_{0}}{Q_{0}}\right)$, the initial stress factor $\left(\frac{P}{2 Q_{0}}\right)$ and also on the direction of propagation denoted by $\left(p_{1}, p_{2}\right)$.

\section{Particular cases:}

Following cases have been discussed to gain more insight information from eq.(10) and eq.(11):

Case I: When $a \rightarrow 0$, i.e., rigidity along vertical direction is constant, eq. (10) reduces to

$$
\left(\frac{c_{1}}{\beta}\right)^{2}=\frac{1}{(1+c y)}\left\{\left(1-\frac{P}{2 Q_{0}}\right) p_{1}^{4}+2\left[\frac{2 N_{0}}{Q_{0}}(1+b y)-1\right] p_{1}^{2} p_{2}^{2}+\left(1+\frac{P}{2 Q_{0}}\right) p_{2}^{4}\right\} .
$$

The velocity of wave along $x$-direction $\left(p_{1}=1, p_{2}=0, c_{1}=c_{11}\right)$, is obtained as

$$
c_{11}^{2}=\beta^{2}\left(1-\frac{P}{2 Q_{0}}\right) \frac{1}{(1+c y)} .
$$

This shows that velocity of wave along $\mathrm{x}$-direction depends on initial stress.

If the medium is free from initial stress, i.e when $P \rightarrow 0$ and $c \rightarrow 0$, the velocity of wave is given by $c_{11}=\beta$. 
Similarly the velocity of propagation along $y$-direction $\left(p_{1}=0, p_{2}=1, c_{1}=c_{22}\right)$, is obtained as

$$
c_{22}^{2}=\beta^{2}\left(1+\frac{P}{2 Q_{0}}\right) \frac{1}{(1+c y)},
$$

It is interesting to note that $\frac{c_{22}^{2}-c_{11}^{2}}{\beta^{2}}=\frac{P}{Q_{0}} \frac{1}{(1+c y)}$, a function of initial stress and density.

It is also observed that if $P>0$, the effect of initial stresses on the body is compressive along $x$-direction and which reduces the velocity of shear wave along $x$-direction while tensile stress increases the velocity of shear wave, where as along $y$-direction shear wave velocity shows the reverse effect.

Case II: When $b \rightarrow 0$, i.e., rigidity along horizontal direction is constant, eq. (10) reduces to

$$
\begin{aligned}
\left(\frac{c_{1}}{\beta}\right)^{2}= & \frac{1}{(1+c y)}\left\{\left[(1+a y)-\frac{P}{2 Q_{0}}\right] p_{1}^{4}+2\left[\frac{2 N_{0}}{Q_{0}}-(1+a y)\right] p_{1}^{2} p_{2}^{2}\right. \\
& \left.+\left[(1+a y)+\frac{P}{2 Q_{0}}\right] p_{2}^{4}\right\} .
\end{aligned}
$$

The velocity of wave along $x$ - direction $\left(p_{1}=1, p_{2}=0, c_{1}=c_{11}\right)$, is given by

$$
c_{11}^{2}=\beta^{2}\left[(1+a y)-\frac{P}{2 Q_{0}}\right] \frac{1}{(1+c y)},
$$

which depends on the depth $y$ and the wave is dispersive.

The velocity along $y$-direction is

$$
c_{22}^{2}=\beta^{2}\left[(1+a y)+\frac{P}{2 Q_{0}}\right] \frac{1}{(1+c y)},
$$

In case of $P>0$, the velocity along $y$-direction may increase considerably at a distance $y$ from free surface and the wave is dispersive.

Case III: When $a \rightarrow 0, b \rightarrow 0$, i.e., the rigidity along horizontal direction is constant but density is linearly varying with depth, eq. (10) transforms to

$$
\left(\frac{c_{1}}{\beta}\right)^{2}=\frac{1}{(1+c y)}\left\{\left(1-\frac{P}{2 Q_{0}}\right) p_{1}^{4}+2\left(\frac{2 N_{0}}{Q_{0}}-1\right) p_{1}^{2} p_{2}^{2}+\left(1+\frac{P}{2 Q_{0}}\right) p_{2}^{4}\right\} .
$$

In the absence of initial stress the velocity of wave is

$$
\left(\frac{c_{1}}{\beta}\right)^{2}=\frac{1}{(1+c y)}\left[1-4\left(1-\frac{N_{0}}{Q_{0}}\right) p_{1}^{2} p_{2}^{2}\right] \text {. }
$$

This shows that velocity $c_{1}^{2}=\frac{\beta^{2}}{(1+c y)}$, in $x$-direction $\left(p_{1}=1, p_{2}=0, c_{1}=c_{11}\right)$, and in

$y$-direction $\left(p_{1}=0, p_{2}=1, c_{1}=c_{22}\right)$ it does not depend on anisotropy. However, in other directions the anisotropy affects the velocity.

For $N_{0}=Q_{0}$, i.e., for isotropic medium with variable density the wave velocity is

$$
\left(\frac{c_{1}}{\beta}\right)^{2}=\frac{p_{1}^{2} p_{2}^{2}}{(1+c y)},
$$

which depends on the direction of propagation.

Case IV : In the absence of initial stress i.e. $P \rightarrow 0$, eq. (10) gives the velocity of wave as 


$$
\left(\frac{c_{1}}{\beta}\right)^{2}=\frac{1}{(1+c y)}\left\{(1+a y) p_{1}^{4}+2\left[2 \frac{N_{0}}{Q_{0}}(1+b y)-(1+a y)\right] p_{1}^{2} p_{2}^{2}+(1+a y) p_{2}^{4}\right\} .
$$

which for $N_{0}=Q_{0}$, takes the form

$$
\left(\frac{c_{1}}{\beta}\right)^{2}=\frac{1}{(1+c y)}\left\{(1+a y) p_{1}^{4}+2[2(1+b y)-(1+a y)] p_{1}^{2} p_{2}^{2}+(1+a y) p_{2}^{4}\right\} .
$$

Along $y$-direction $\left(p_{1}=0, p_{2}=1, c_{1}=c_{22}\right)$,

$$
\left(\frac{c_{22}}{\beta}\right)^{2}=\frac{1+a y}{1+c y} .
$$

Along $x$-direction $\left(p_{1}=1, p_{2}=0, c_{1}=c_{11}\right)$,

$$
\left(\frac{c_{11}}{\beta}\right)^{2}=\frac{1+a y}{1+c y} .
$$

The wave is dispersive and velocities are same in two direction $x$ and $y$.

Case $V$ : When $a \rightarrow 0$, i.e., rigidity along vertical direction is constant, eq. (11) transforms to

$$
\left(\frac{c_{1}}{\beta}\right)^{2}=2\left(\frac{2 N_{0}}{Q_{0}} \frac{b}{c}\right) p_{1}^{2}
$$

this shows that velocity of shear wave is always damped.

The velocity of wave along $x$-direction $\left(p_{1}=1, p_{2}=0, c_{1}=c_{11}\right)$, is obtained as

$$
\left(\frac{c_{11}}{\beta}\right)^{2}=2\left(\frac{2 N_{0}}{Q_{0}} \frac{b}{c}\right)
$$

this shows that actual wave velocity in $x$-direction is damped by $2\left(\frac{2 N_{0}}{Q_{0}} \frac{b}{c}\right)$, whereas, no damping takes place along $y$-direction $\left(p_{1}=0, p_{2}=1, c_{1}=c_{22}\right)$.

Case VI : When $b \rightarrow 0$, i.e. rigidity along horizontal direction is constant, eq. (11) reduces to

$$
\left(\frac{c_{1}}{\beta}\right)^{2}=2\left(-\frac{a}{c}\right) p_{1}^{2}+2\left(\frac{a}{c}\right) p_{2}^{2} .
$$

The velocity of wave along $x$ - direction $\left(p_{1}=1, p_{2}=0, c_{1}=c_{11}\right)$, is given by

$$
\left(\frac{c_{11}}{\beta}\right)^{2}=2\left(-\frac{a}{c}\right) \text {. }
$$

Existence of negative sign shows that damping does not takes place along $x$-direction for $b \rightarrow 0$, whereas damping of magnitude $\left(\frac{2 a}{c}\right)$, takes place along $\mathrm{y}$-direction.

Case VII: When $a \rightarrow 0, b \rightarrow 0$, i.e. rigidity along horizontal direction is constant but density varying linearly with depth, eq.

(11) gives $\left(\frac{c_{1}}{\beta}\right)^{2}=0$, i.e. no damping takes place for $a \rightarrow 0, b \rightarrow 0$. 


\section{Numerical calculation}

To get numerical information on the velocity of shear wave in the non-homogeneous initially stressed medium, the equation (10) is non-dimensionalized as

$$
\begin{aligned}
\left(\frac{c_{1}}{\beta}\right)^{2}= & \frac{1}{\left(1+\frac{c}{b} b y\right)}\left\{\left(1+\frac{a}{b} b y-\frac{P}{2 Q_{0}}\right) p_{1}^{4}+2\left[\frac{2 N_{0}}{Q_{0}}(1+b y)-\left(1+\frac{a}{b} b y\right)\right] p_{1}^{2} p_{2}^{2}\right. \\
& \left.+\left(1+\frac{a}{b} b y+\frac{P}{2 Q_{0}}\right) p_{2}^{4}\right\} .
\end{aligned}
$$

The numerical values of $\frac{c_{1}}{\beta}$ has been calculated for different values of $\frac{a}{b}, \frac{N_{0}}{Q_{0}}, p_{1}, p_{2}$ and $\frac{P}{2 Q_{0}}$, and the results are presented in Figures 1 through 6.

Figure-1 gives the variation in velocities of shear wave in the direction of $\theta=30^{\circ}$ and $60^{\circ}$ with $x$-axis at different depth and different values of density parameter $\left(\frac{c}{b}\right)=0.7,0.8$, and 0.9 for $\frac{a}{b}=4.0, \frac{P}{2 Q_{0}}=0.5$, and $\frac{N_{0}}{Q_{0}}=2.5$. The velocity of the wave increases as depth increases.

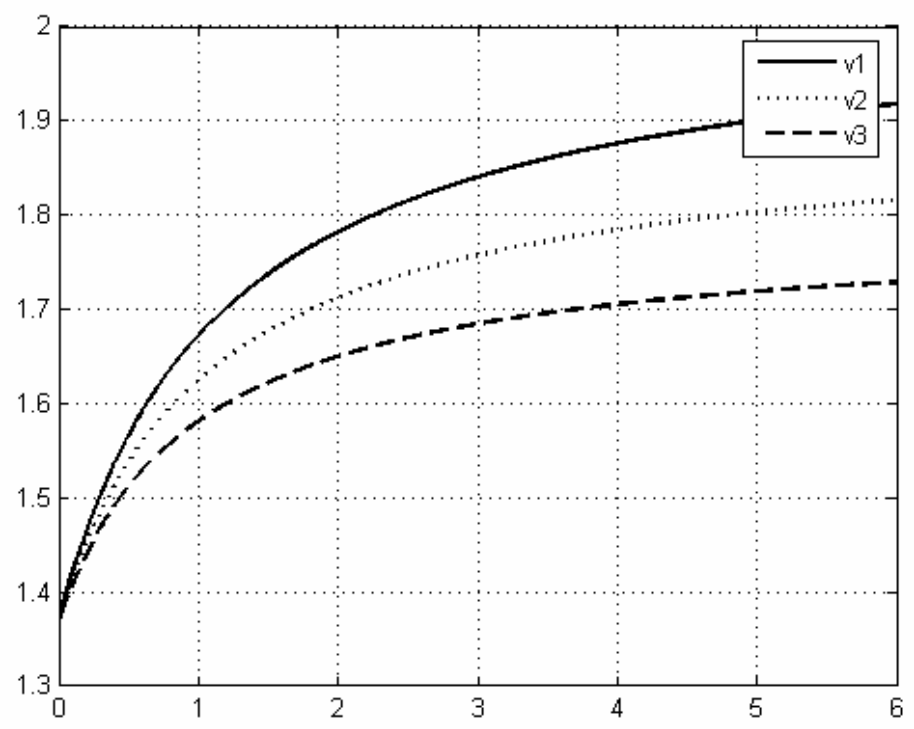

Figure 1a $\left(\theta=30^{0}\right)$ : by vs. $\frac{c_{1}}{\beta}$ for $v 1:\left(\frac{c}{b}\right)=0.7, v 2:\left(\frac{c}{b}\right)=0.8, v 3:\left(\frac{c}{b}\right)=0.9$. 


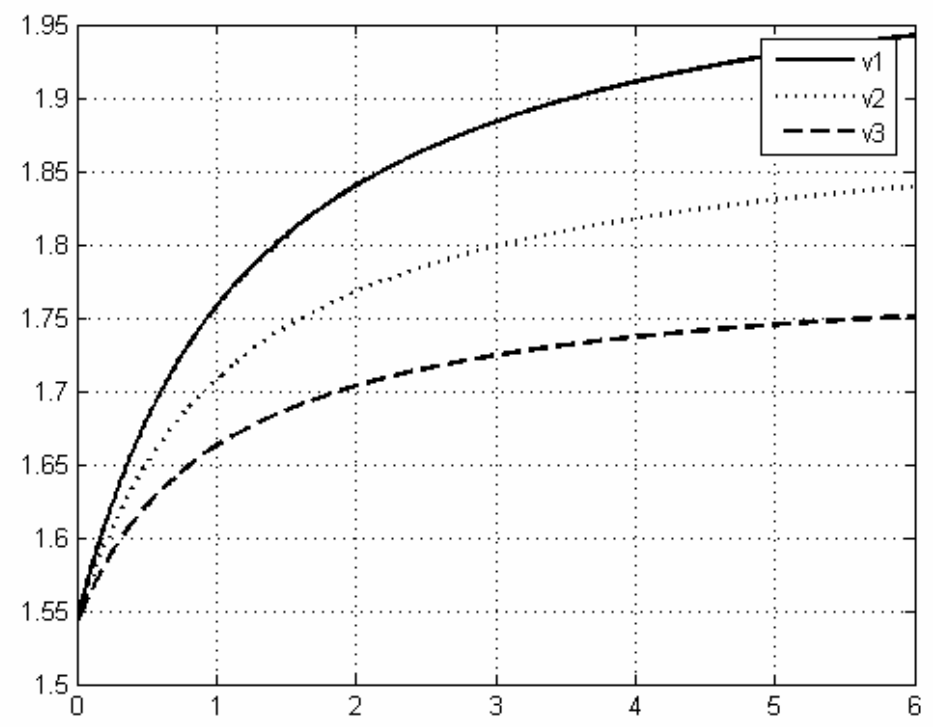

Figure $1 \mathrm{~b}\left(\theta=60^{0}\right)$ : by vs. $\frac{c_{1}}{\beta}$ for $v 1:\left(\frac{c}{b}\right)=0.7, v 2:\left(\frac{c}{b}\right)=0.8, v 3:\left(\frac{c}{b}\right)=0.9$.

Figure. 2 gives the variation in velocities of shear wave in the direction of $\theta=30^{\circ}$ and $60^{\circ}$ with $x$-axis at different depth and different values of $\frac{a}{b}$ when $\frac{c}{b}=0.8, \frac{P}{2 Q_{0}}=0.5$, and $\frac{N_{0}}{Q_{0}}=2.5$. The velocity of the wave increases as depth increases.

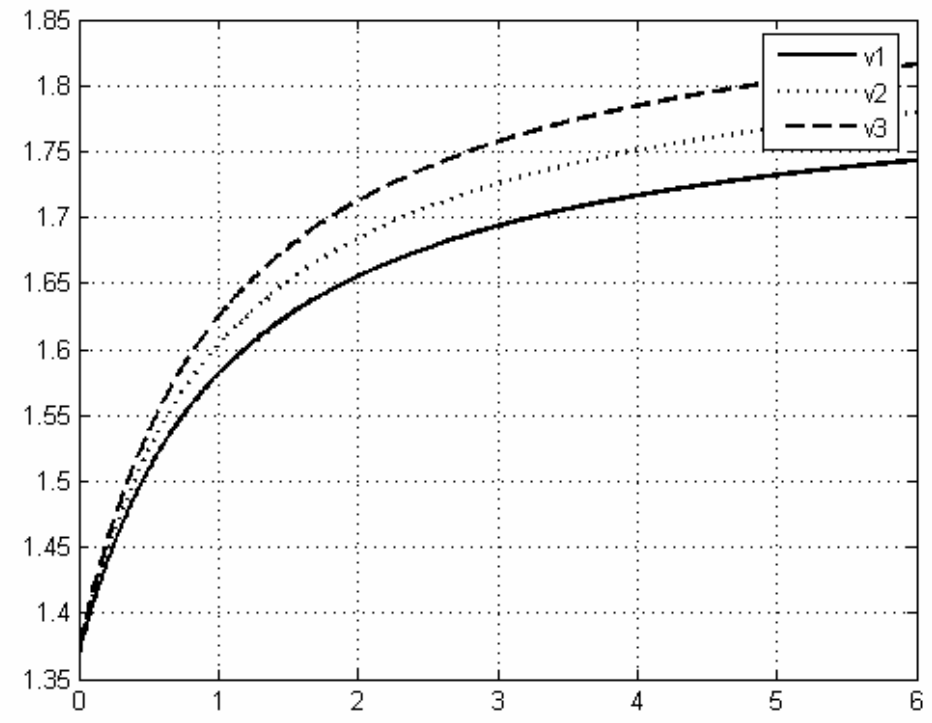

Figure 2a $\left(\theta=30^{\circ}\right)$ : by vs. $\frac{c_{1}}{\beta}$ for $v 1:\left(\frac{a}{b}\right)=3.0, v 2:\left(\frac{a}{b}\right)=3.5, v 3:\left(\frac{a}{b}\right)=4.0$. 


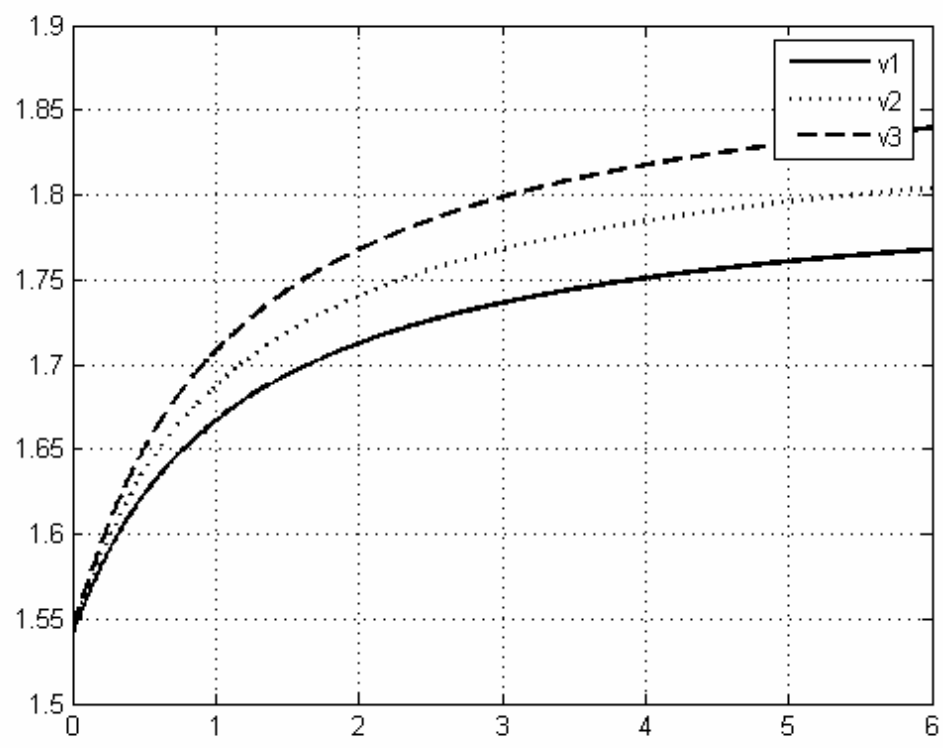

Figure $2 \mathrm{~b}\left(\theta=60^{0}\right)$ : by vs. $\frac{c_{1}}{\beta}$ for $v 1:\left(\frac{a}{b}\right)=3.0, v 2:\left(\frac{a}{b}\right)=3.5, v 3:\left(\frac{a}{b}\right)=4.0$.

Figure. 3 gives the variation in velocities of shear wave in the direction of $\theta=30^{\circ}$ and $60^{\circ}$ with $x$-axis at different depth and different values of $\frac{N_{0}}{Q_{0}}$ when $\frac{c}{b}=0.8, \frac{P}{2 Q_{0}}=0.5$, and $\frac{a}{b}=4.0$. Figure.3 gives the information of variation of velocity for different values of anisotropic factor and reflects that with the increase in the values of $\frac{N_{0}}{Q_{0}}$, the velocity of shear wave increases.

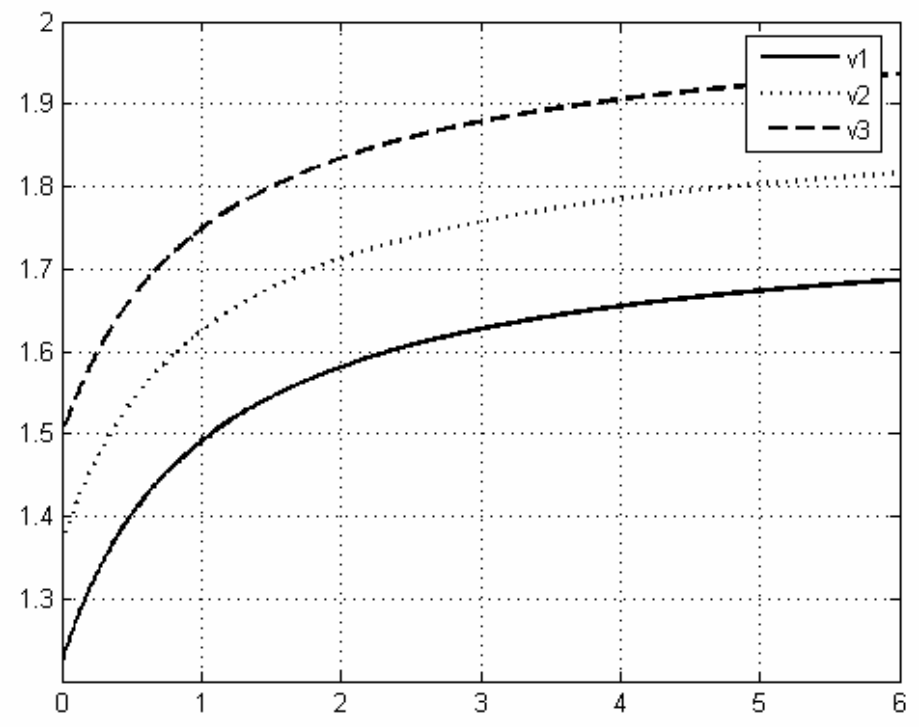

Figure 3a $\left(\theta=30^{\circ}\right)$ : by vs. $\frac{c_{1}}{\beta}$ for $v 1$ : $\left(\frac{N_{0}}{Q_{0}}\right)=2.0, v 2$ : $\left(\frac{N_{0}}{Q_{0}}\right)=2.5, v 3$ : $\left(\frac{N_{0}}{Q_{0}}\right)=3.0$. 


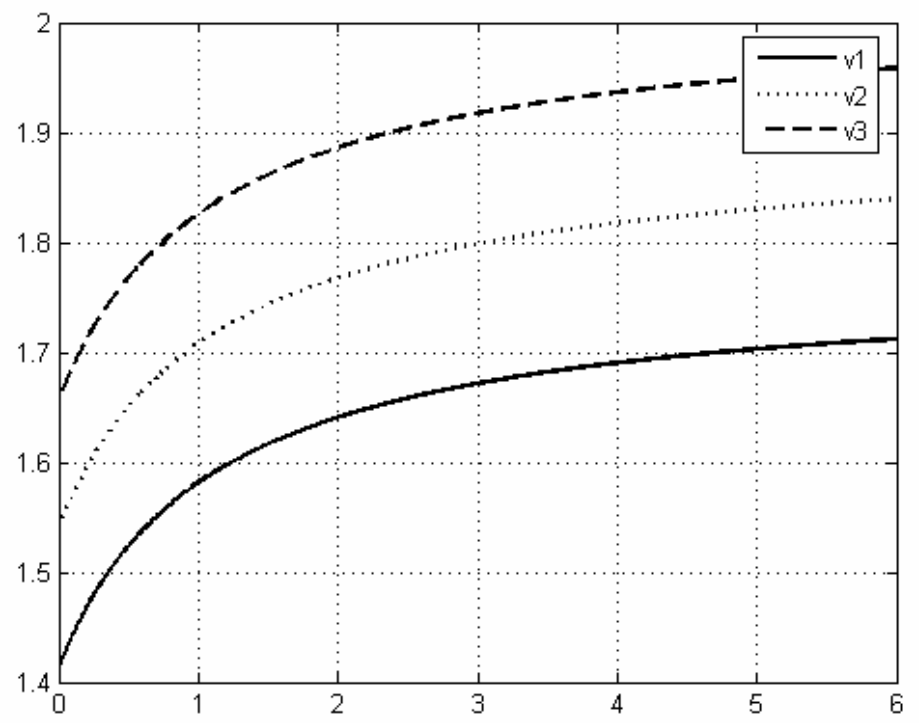

Figure $3 b\left(\theta=60^{\circ}\right)$ : by vs. $\frac{c_{1}}{\beta}$ for $v 1:\left(\frac{N_{0}}{Q_{0}}\right)=2.0, v 2:\left(\frac{N_{0}}{Q_{0}}\right)=2.5, v 3:\left(\frac{N_{0}}{Q_{0}}\right)=3.0$.

Fig.4 gives the variation in velocities of shear wave in the direction of $\theta=30^{\circ}$ and $60^{\circ}$ with $x$-axis at different depth and different values initial stress parameter $\frac{P}{2 Q_{0}}$ when $\frac{c}{b}=0.8, \frac{N_{0}}{Q_{0}}=2.5$, and $\frac{a}{b}=4.0$. The velocity of the wave increases as depth increases.

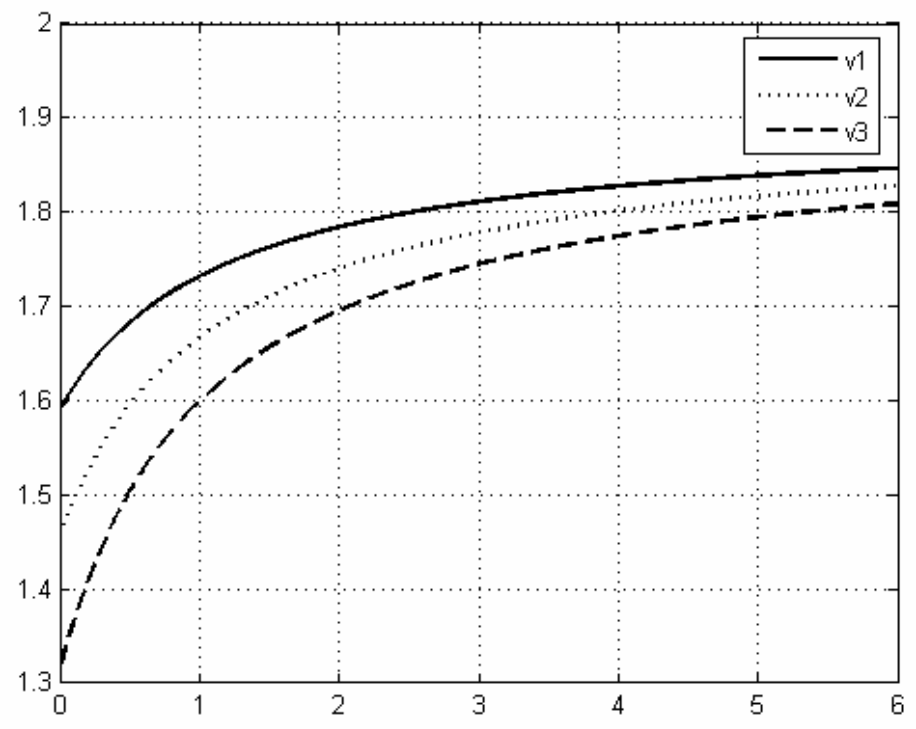

Figure $4 \mathrm{a}\left(\theta=30^{0}\right)$ : by vs. $\frac{c_{1}}{\beta}$ for $v 1:\left(\frac{P}{2 Q_{0}}\right)=-0.8, v 2:\left(\frac{P}{2 Q_{0}}\right)=0.0, v 3:\left(\frac{P}{2 Q_{0}}\right)=0.8$. 


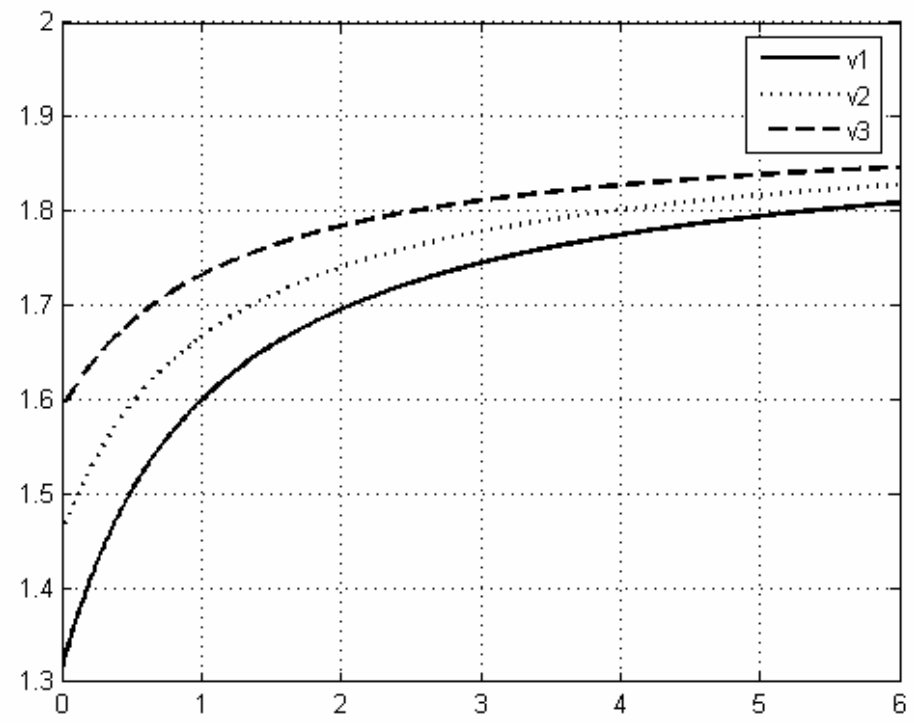

Figure $4 \mathrm{~b}\left(\theta=60^{0}\right)$ : by vs. $\frac{C_{1}}{\beta}$ for $v 1:\left(\frac{P}{2 Q_{0}}\right)=-0.8, v 2:\left(\frac{P}{2 Q_{0}}\right)=0.0, v 3:\left(\frac{P}{2 Q_{0}}\right)=0.8$.

Figure.5 gives the velocity of shear wave in an anisotropic initially stressed homogeneous medium for different values of initial stress parameter $\frac{P}{2 Q_{0}}$ and $\frac{N_{0}}{Q_{0}}$ with $\frac{P}{2 Q_{0}}=0.5, \frac{c}{b}=0.8, \frac{N_{0}}{Q_{0}}=2.5$, and $\frac{a}{b}=4.0$.

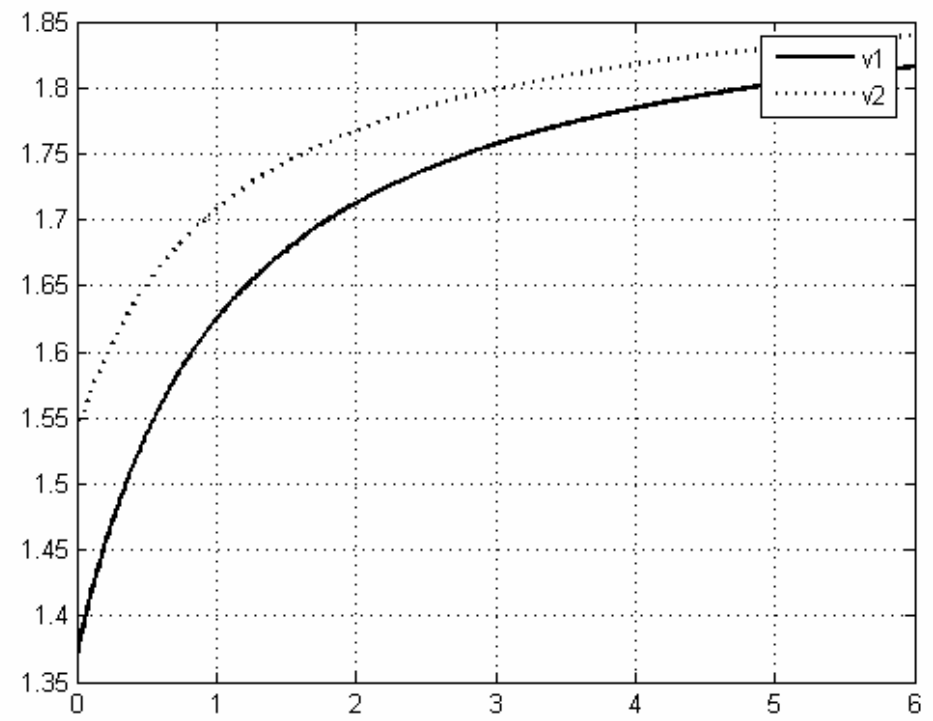

Figure 5: by vs. $\frac{C_{1}}{\beta}$ for $v 1: \theta=30^{\circ}, v 2: \theta=60^{\circ}$

Figure.6 shows the variation of velocity of shear wave with respect to direction of propagation in non-homogeneous anisotropic initially stressed medium with $\frac{P}{2 Q_{0}}=0.5, \frac{c}{b}=0.8, \frac{N_{0}}{Q_{0}}=2.5, \frac{a}{b}=4.0$, and by $=2.0$; 


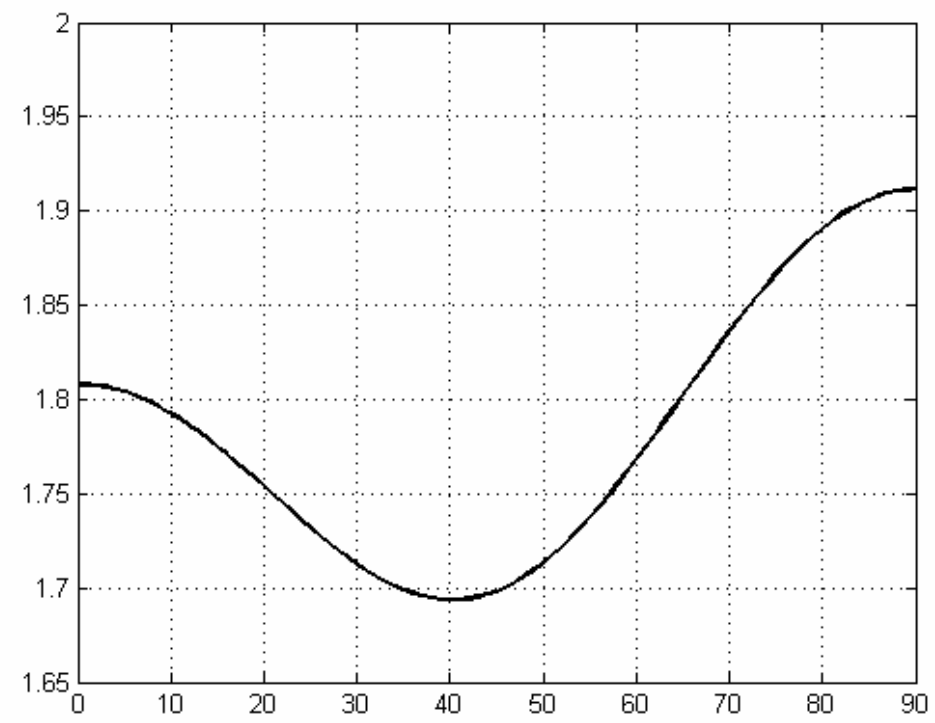

Figure 6: $\theta$ (in degrees) vs. $\frac{C_{1}}{\beta}$.

\section{Conclusion}

From equation (10) it is concluded that:

1. When rigidity along vertical direction is constant then shear wave velocity is influenced by initial stress. We have derived the velocity of wave in both $x$ and $y$ direction and we have seen both depend on initial stress. Compressive initial stress reduces the velocity of shear wave along $x$-direction while tensile stress increases. Shear wave velocity shows the reverse effect along $y$-direction.

2. If rigidity along horizontal direction is constant then shear wave velocity exists and we have obtained the velocity equation in both $\mathrm{x}$ and y direction. In case $P>0$, the velocity along $x$-direction may decrease considerably and the velocity along $y$ direction may increase considerably.

3. When the rigidity along horizontal direction is constant but density is linearly varying with depth then also shear wave velocity is still influenced by initial stress and in absence of initial stress the velocity also exist. The velocity of wave in $x$ direction and $y$-direction does not depend on anisotropy. However, in other directions the anisotropy affects the velocity. We have also observed for isotropic medium with variable density the velocity depends on the direction of propagation.

4. In the absence of initial stress the shear wave velocity is still available. It also exists in isotropic medium. But in this case velocities are same in two direction $x$ and $y$.

From equation (11) it is concluded that:

1. If rigidity along vertical direction is constant then the velocity of shear wave is always damped. We have obtained the velocity equation of wave along $x$-direction and it is also damped whereas no damping takes place along $y$-direction.

2. When rigidity along horizontal direction is constant then the velocity of shear wave is damped and in this case the damping does not take place along $x$-direction whereas damping takes place along $y$-direction.

3. When rigidity along horizontal direction is constant but density varying linearly with depth then no damping takes place.

Thus it seen that the anisotropy, non-homogeneity , the initial stresses , the direction of propagation and the depth (in case of nonhomogeneous medium) have considerable effect in the velocity of propagation of shear wave and attracts the attention of earth scientists in their work.

\section{References}

Achenbach, J.D. 1973. Wave propagation in elastic solids, North Holland Publishing Comp., New York.

Addy, S. K. and Chakraborty ,N. R., 2005. Rayleigh waves in a viscoelastic half-space under initial hydrostatic stress in presence of the temperature field. International Journal of Mathematics and Mathematical Sciences, Vol. 24, pp. 3883-3894

Bath, M. A., 1968. Mathematical Aspects of Seismology, Elsevier Publishing Comp., New York. 
Biot, M.A., 1940. The influence of initial stress on elastic waves, Journal of App. Phy., Vol.2, p. 522

Biot, M.A., 1965. Mechanics of incremental deformations, John Wiley and Sons Inc, New York.

Chattopadhyay, A., Gupta, S., Singh, A.K. and Sahu, S.A., 2009. Propagation of shear waves in an irregular magnetoelastic monoclinic layer sandwiched between two isotropic half-spaces, International Journal of Engineering, Science and Technology, Vol. 1, No. 1, pp. 228-244.

Chattopadhyay, A., Gupta, S., Sharma, V.K. and Kumari, P., 2010. Effects of irregularity and anisotropy on the propagation of shear waves, International Journal of Engineering, Science and Technology, Vol. 2, No. 1, pp. 116-126.

Duan1, B. and Oglesby, D.D., 2006. Heterogeneous fault stresses from previous earthquakes and the effect on dynamics of parallel strike-slip faults. Journal of Geophysical Research, Vol. 111, B05309.

Duan1, B. and Oglesby, D.D., 2007. Nonuniform prestress from prior earthquakes and the effect on dynamics of branched fault systems, Journal of Geophysical Research, Vol. 112, B05308.

Dey, S. and De, P. K. 2009. Edge wave propagation in an incompressible anisotropic initially stressed plate of finite thickness, International Journal of Computational Cognition, Vol. 7, No. 3, pp. 55-60.

Ewing, W. M., Jardetzky, W. S. and Press, F. 1957. Elastic waves in layered media, McGraw Hill Book Comp., New York.

Huber, A., 2010. The physical meaning of a nonlinear evolution equation of the fourth order relating to locally and non-locally supercritical waves, International Journal of Engineering, Science and Technology, Vol. 2, No. 1, pp. 70-79.

Kappus, R. 1939. Zeitschr. A ngew Math.Mech, Vol. 19, No. 5, p. 27.

Liu, Y. and Wei , X. C. 2008. Propagation characteristics of converted refracted wave and its application in static correction of converted wave, Science in China Series D: Earth Sciences, Vol. 51, No. 2, pp. 226-232.

Murnaghan, F. D. 1951. Finite Deformation of an Elastic Solids, John Willey and Sons, New York.

Moczo, P., Robertsson, J.O.A., and Eisner, L., 2007. The Finite-difference time-domain method for modeling of seismic wave propagation, Advances in Geophysics, Vol. 48, pp. 421-516.

Qian ,Z., Jin, F., Kishimoto. K., and Wang, Z., 2004. Effect of initial stress on the propagation behavior of SH-waves in multilayered piezoelectric composite structures, Sensors and Actuators A: Physical, Vol. 112, No. 2-3, pp. 368-375.

Selim. M.M. and Ahmed. M.K., 2006. Propagation and attenuation of seismic body waves in dissipative medium under initial and couple stresses, Applied Mathematics and Computation, Vol. 182, No. 2, pp. 1064-1074.

Sharma M. D. 2005. Effect of initial stress on the propagation of plane waves in a general anisotropic poroelastic medium, Journal of Geophysical Research, Vol. 110, No. B11, pp. B11307.1-B11307.14

Sharma M. D. and Garg N., 2006. Wave velocities in a pre-stressed anisotropic elastic medium, Journal of Earth System Science, Vol. 115, No. 2, pp. 257-265.

Zhang, J., Shen, Y.P. and Du J.K., 2008. The effect of inhomogeneous initial stress on Love wave propagation in layered magneto-electro-elastic structures, Smart Mater. Struct. Vol. 17, No. 025026 (9pp)

Zhou, Y. and Chen, Y., 2005. Influence of seismic cyclic loading history on small strain shear modulus of saturated sands. Soil Dynamics and Earthquake Engineering, Vol. 25, No. 5, pp. 341-353.

\section{Biographical notes}

Dr. Shishir Gupta is an Associate Professor in the Department of Applied mathematics, Indian School of Mines, Dhanbad. A Gold Medalist from Ranchi University, he has had a brilliant career. He has more than 21 years of teaching experience at undergraduate and postgraduate levels in Indian School of Mines, Dhanbad. He possesses experience of guiding students of MPhil and PhD. He has published more than 45 papers in International/National journals/Proceedings. He has served as reviewer in renowned International/ National books and journals. He has also carried out several sponsored research projects.

Mr. Santimoy Kundu is a Junior Research Fellow (JRF) in the Department of Applied Mathematics, Indian School of Mines (ISM), Dhanbad, Jharkhand, India. He is pursuing his PhD under the supervision Dr. Shishir Gupta of ISM in the field of Theoretical Seismology. He did his B.Sc. from University of Calcutta, Kolkata in 2001 and then switched to ISM to earn M.Sc. in Maths \& Computing (2004) and MPhil in Applied Mathematics (2006).

A.K. Verma is an Endowed University Professor of Mathematics at Hampton University, Hampton, Virginia, USA. In his teaching caereer of over twenty-five years he has authored/co-authored research papers in fluid mechanics, controlled thermo-nuclear fusion and education technology. Dr. Verma had served as director and consultant for state and federal projects, and reviewed federal proposals. He has earned several accolades at institution, regional, state and national levels for his quality teaching and effective use of technology in instruction.

R. Verma is an Associate Professor in the Department of Mathematics, Norfolk State University, Norfolk, Virginia. She earned her Ph.D. from Indian Institute of Technology, Kharagpur. Dr. Verma has taught mathematics in traditional way and online to technical and non-technical students for over twenty-years. Her research focuses on the use of integral equations in crack problems of elasticity. She has several publications in this field in international journals. Dr. Verma has also been involved in training school teachers to incorporate new teaching methods using technology.

Received December 2009

Accepted March 2010

Final acceptance in revised form April 2010 